\title{
Structural Characterization of the Complex between Hen Egg-White Lysozyme and Zr(IV)-Substituted Keggin Polyoxometalate as Artificial Protease
}

\author{
Annelies Sap ${ }^{[a]}$, Elke De Zitter ${ }^{[a]}$, Luc Van Meervelt ${ }^{[a]}$, Tatjana N. Parac-Vogt ${ }^{\star[a]}$
}

[a] A. Sap, E. De Zitter, Prof. L. Van Meervelt, Prof. T. N. Parac-Vogt

Department of Chemistry

KU Leuven

Celestijnenlaan 200F box 2404, 3001 Leuven (Heverlee), Belgium

E-mail: tatjana.vogt@chem.kuleuven.be

\begin{abstract}
Successful co-crystallization of a non-covalent complex between hen egg-white lysozyme (HEWL) and the monomeric $\mathrm{Zr}(\mathrm{IV})$-substituted Keggin polyoxometalate $(\mathrm{POM})(\mathrm{Zr} 1-\mathrm{K} 1),\left(\mathrm{Et}_{2} \mathrm{NH}_{2}\right)_{3}\left[\mathrm{Zr}\left(\mathrm{PW}_{11} \mathrm{O}_{39}\right)\right](1)$, has been achieved and its single crystal $\mathrm{x}$-ray structure has been determined. The dimeric $\mathrm{Zr}(\mathrm{IV})$-substituted Keggin-type polyoxometalate (Zr1-K2), $\left(\mathrm{Et}_{2} \mathrm{NH}_{2}\right)_{10}\left[\mathrm{Zr}\left(\mathrm{PW}_{11} \mathrm{O}_{39}\right)_{2}\right](2)$ has been previously shown to exhibit remarkable selectivity towards HEWL hydrolysis. The reported $x$-ray structure shows that the hydrolytically active $\mathrm{Zr}(\mathrm{IV})$-substituted Keggin POM exists as a monomeric species. Prior to hydrolysis, this monomer interacts with HEWL in the vicinity of the previously identified cleavage sites found at Trp28-Val29 and Asn44-Arg45, via water-mediated $\mathrm{H}$-bonding and electrostatic interactions. Three binding sites are observed at the interface of the negatively charged Keggin POM and the positively charged regions of HEWL: at i) Gly16, Tyr20 and Arg21, ii) Asn44, Arg45 and Asn46, and iii) Arg128.
\end{abstract}

The structural and compositional variety of polyoxometalates with respect to size, shape, charge density, solubility, acid strength, and redox potential make them attractive chemical agents in many scientific disciplines such as catalysis, material science and medicine. ${ }^{[1]}$ A detailed investigation of the biological activity of POMs has led to the discovery of their antibacterial, antiviral and anticancer properties. ${ }^{[\mathrm{b}]}$ The large, three-dimensional structure and high negative charge allow POMs to bind to positively charged surface patches of proteins, presumably via electrostatic interactions ${ }^{[2]}$ This affinity of POMs towards protein surfaces has inspired us to develop a novel class of artificial metalloproteases by combining the enzyme-like recognition ability of the polyoxotungstate surface with an embedded Lewis acidic metal for hydrolytic activity. We demonstrated the first example of selective protein hydrolysis by a POM complex, in which hen egg-white lysozyme (HEWL) was selectively cleaved by $\mathrm{Ce}(\mathrm{IV})$ - and $\mathrm{Zr}(\mathrm{IV})$-substituted Keggin POMs. ${ }^{[3]}$ Several other proteins, including insulin chain $\mathrm{B}^{[4]}$ and human serum albumin $(\mathrm{HSA})^{[5]}$ have also been selectively and efficiently hydrolyzed by different $\mathrm{Zr}(\mathrm{IV})$-substituted POMs. This novel reactivity of POMs is especially interesting as the peptide bond is extremely stable towards hydrolysis, with a half-life for the uncatalyzed reaction of up to 600 years under physiological conditions. ${ }^{[6]}$ Nevertheless, selective hydrolysis of proteins is one of the most required procedures in biotechnology and proteomics, and the few existing chemical reagents often require harsh reaction conditions and tend to cleave proteins with partial selectivity and low yields. ${ }^{[7]}$ In this respect, metal-substituted POMs are an attractive alternative because they cleave proteins in a selective manner and operate under mild reaction conditions of $\mathrm{pH}$ and temperature.

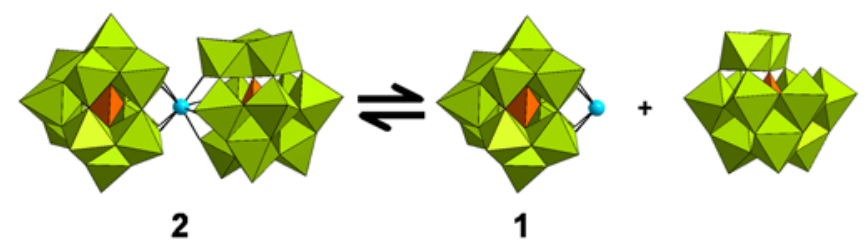

Scheme 1: Equilibrium of 1:2 species (2) (left) with 1:1 species (1) (right) and a-lacunary species (right) of the Zr(IV)-substituted Keggin POM. $\mathrm{WO}_{6}$ octahedrons are represented in green, $\mathrm{PO}_{4}$ tetrahedrons in orange, and $\mathrm{Z} \mathrm{r}^{\mathrm{IV}}$ in cyan.

In a previous study we have shown that $\mathrm{Ce}(\mathrm{IV})$ and $\mathrm{Zr}(\mathrm{IV})$-substituted Keggin POMs, $\left[\mathrm{Ce}\left(\mathrm{\alpha}-\mathrm{PW} \mathrm{W}_{11} \mathrm{O}_{39}\right)_{2}\right]^{10-}$ and $[\mathrm{Zr}(\mathrm{\alpha}-$ $\left.\left.\mathrm{PW}_{11} \mathrm{O}_{39}\right)_{2}\right]^{10-}$ selectively hydrolyzed HEWL under physiological $\mathrm{pH}$ at two cleavage sites, Trp28-Val29 and Asn44-Arg45. [3] Different spectroscopic techniques have been used to demonstrate that interaction between HEWL and POMs took place in solution ${ }^{[3]}$, however they were unable to reveal the exact nature of the protein/POM complex. Furthermore, the most intriguing question that has not been answered so far, concerns the nature of the active POM catalyst responsible for the hydrolysis of the peptide bond. The 1:2 Zr(IV)-substituted Keggin POM has so far been characterized only as a dimeric 1:2 complex (2) (see Figure S1 and Figure S2), both in solution and in the solid state. ${ }^{[8]}$ It has been suggested that in solution, the dimeric $1: 2$ species (2) and the monomeric 1:1 species (1) exist in equilibrium ( 
Scheme 1). ${ }^{[9]}$ However the $1: 1$ species was proposed to exist only in very acidic solutions with $\mathrm{pH}<1,{ }^{[10]}$ and to the best of our knowledge, no $x$-ray structural proof for the existence of 1 has been given so far.

The puzzling fact is that from the catalytic point of view, $\mathbf{2}$ is a highly unlikely catalyst, as the coordination sphere of $\mathrm{Zr}(\mathrm{IV})$ is fully saturated by coordination to oxygen atoms of the POM. Effective peptide bond hydrolysis requires interaction between the amide carbonyl and the metal ion, leading to polarization of the carbonyl group. Therefore the 1:1 species (1) had earlier been proposed to be an active catalyst, and a hypothesis was made that an interaction of 2 with HEWL would induce partial dissociation of the dimeric species (2) into the 1:1 complex (1), with the latter acting as a catalyst.[3] However until now, no evidence was found to support this hypothesis and the lack of any experimental evidence for the existence of the 1:1 complex prevented full understanding of the mechanism of this novel POM reactivity towards protein hydrolysis.

Herein, we report for the first time on the successful co-crystallization of a 1:1 $\mathrm{Zr}(\mathrm{IV})$-substituted Keggin-type POM, $\left(\mathrm{Et}_{2} \mathrm{NH}_{2}\right)_{3}\left[\mathrm{Zr}\left(\mathrm{PW}_{11} \mathrm{O}_{39}\right)\right](1)$, with HEWL. The structure was determined with a resolution of $1.38 \AA$, and interestingly, although HEWL was mixed with the synthesized 1:2 complex $\left(\mathrm{Et}_{2} \mathrm{NH}_{2}\right)_{10}\left[\mathrm{Zr}\left(\mathrm{PW}_{11} \mathrm{O}_{39}\right)_{2}\right](2)$, the crystal structure showed exclusively the presence of 1:1 species (1) interacting with HEWL.

The overall fold of HEWL in our co-crystal structure is very similar to that seen in other structures (e.g. $C_{\alpha}$ RMSD value with PDB ID $1 \mathrm{LYZ}^{[11]}$ and $193 \mathrm{~L}^{[12]}$ are $0.53 \AA$ and $0.31 \AA$, respectively). ${ }^{[13]}$ Moreover, the co-crystal of $\mathrm{HEWL}-1: 1$ Zr-Keggin POM (1), described in this paper (Figure 1), has the same $\mathrm{P} 4{ }_{3}{ }_{2}{ }_{1}$ space group as seen in native HEWL crystal structures. In these native HEWL crystal structures, voids are present on top of the two-fold axis between four protein monomers. However in our structure, these voids, with an accessible volume of $1461 \AA^{3}$, are filled easily with the monomeric 1:1 species of the Zr-Keggin POM, (1) (Figure 1), while the 1:2 complex (2) was not observed. The 1:1 ZrKeggin POM (1) has an occupancy of $44 \%$. This low occupancy can be explained by precipitation that was observed at the 1:1 molar ratio of Zr-Keggin POM : HEWL used in the crystallization drop (see Figure S3). The actual Zr-Keggin concentration is therefore lower and a $100 \%$ occupancy can therefore never be reached in this specific co-crystal structure. The x-ray data of the co-crystal of HEWL - 1:1 Zr-Keggin POM (1) are given in Table S1.

In protein crystallography, POMs have been often used in crystal soaking experiments for phasing purposes. ${ }^{[14]}$ Recently $\mathrm{abPPO}_{4}$ protein was crystallized using the $\mathrm{Na}_{6}\left[\mathrm{TeW}_{6} \mathrm{O}_{24}\right] .22 \mathrm{H}_{2} \mathrm{O}$ as co-crystallization agent. ${ }^{[15]}$ Also, ribosome D50S crystals were soaked with a Keggin-type POM salt, $\mathrm{K}_{5} \mathrm{H}\left[\mathrm{PW}_{12} \mathrm{O}_{40}\right] .12 \mathrm{H}_{2} \mathrm{O} .^{[16]}$ In only a few cases, a specific POM binding mode to proteins has been identified such as for the polyoxotungstate clusters embedded within the cavity of a Mo/W-storage protein ${ }^{[17]}$ and for the organometallic pro-drug ALF186-HEWL complex. During the crystallization of ALF186 with HEWL, HEWL crystals were soaked into a Mo-containing Keggin POM solution. In this soaking method, the Keggin POM can only enter channels and voids of the established HEWL crystal. Our co-crystallization method of the $\mathrm{Zr}$ (IV)substituted Keggin POM to HEWL however does not have this limitation as POM binding could precede crystallization in solution. Identical interaction sites are however observed for both crystal structures, ALF186-HEWL and 1-HEWL. This demonstrates that the regioselective binding is a result of a strong POM-directed binding.

The position of the $\mathrm{Zr}$ site in our co-crystal structure has been accurately determined, and is strongly supported by the electron density map shown in Figure S4. After an elaborated search, a water molecule with weak electron density that is coordinated to the zirconium atom of 1 was also observed (see Figure S5). Furthermore, the crystal structure is consistent with the reported hydrolysis results, as it indeed shows that the POM-directed binding occurs in the vicinity of the two cleavage sites, Trp28-Val29 and Asn44-Arg45. As this co-crystal structure represents a static binding, conformational changes preceding the dynamic hydrolysis process cannot be excluded. Therefore, there is still a possibility for $\mathrm{Zr}(\mathrm{IV})$ to be directed towards HEWL to generate a weaker metal-directed binding. 


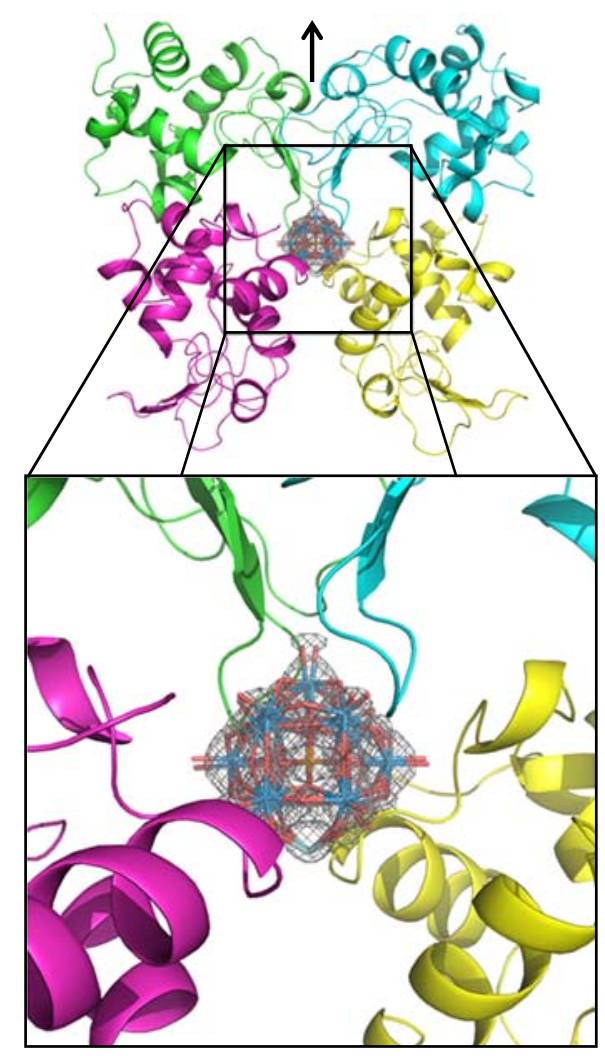

Figure 1: Four HEWL molecules (shown as cartoon in different colors) are surrounding 1 on top of a two-fold axis (shown by the arrow). The inset shows a superposition of the two symmetry related POMs, each with an occupancy of $22 \%$ in the 2 Fo-Fc electron density map contoured at 1.0 $\sigma .1$ is binding to Asn44, Arg45 and Asn46 of two neighboring HEWL molecules (in cyan and green) and to Tyr20 and Arg21 of two other HEWL molecules (in magenta and yellow) ${ }^{[18]}$ The interaction with Arg128 is not shown here for reasons of clarity.

Previous solution NMR interaction studies indicated that several amino acids in the vicinity of two cleavage sites are affected by the addition of POMs. ${ }^{[3]}$ The first hydrolysis site was observed in the $\alpha$-helical region at Trp28-Val29 peptide bond and indeed, Figure 2a demonstrates POM binding to Gly16, Tyr20 and Arg21 residues, which are in close proximity to this cleavage site (Table S2). The electrostatic potential surface, shown in Figure $2 b$, displays the electrostatic interaction between the negatively charged Keggin POM and the positively charged region in proximity of the Trp28-Val29 cleavage site. In addition, in the vicinity of the second cleavage site (Asn44-Arg45) the crystal structure clearly shows a layer of water molecules connecting the Keggin POM to Asn44, Arg45, and Asn46 through several H-bonds (Figure 2c, Table S3). The negatively charged Keggin POM electrostatically interacts with HEWL next to the cleavage site, Asn44Arg45, at the end of $\beta$-sheet 1 (Thr43-Asn46, Figure 2d). Another water-mediated interaction site is observed between the terminal oxygen atoms of 1 and Arg128 of HEWL (Figure 2e,f and Table S4). This is in accordance with NMR results that showed resonance shifts for arginine side chains in the presence of POM. ${ }^{[6]}$ The high negative charge of the Keggin POM (-14) seems to be the main driving force for the electrostatic interactions between the Keggin POM and arginine and lysine side chains of HEWL. 


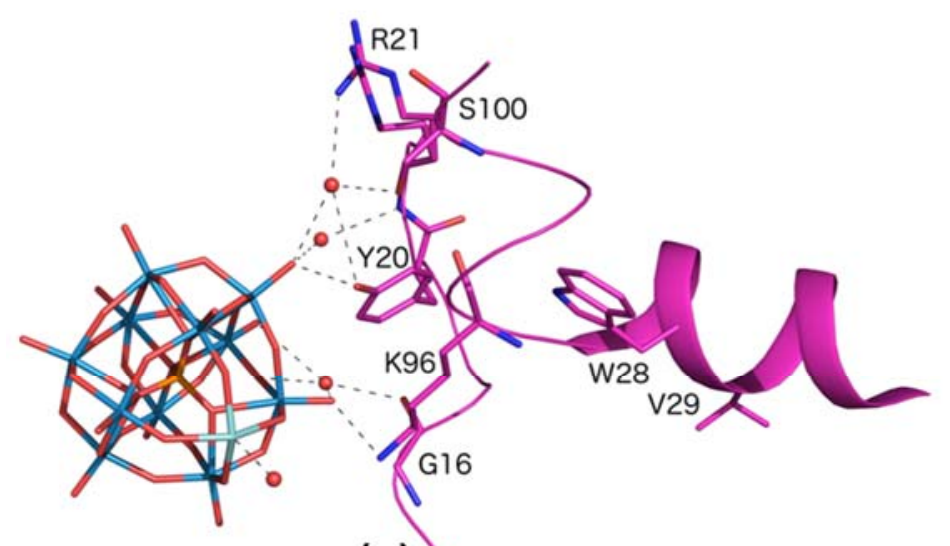

(a)

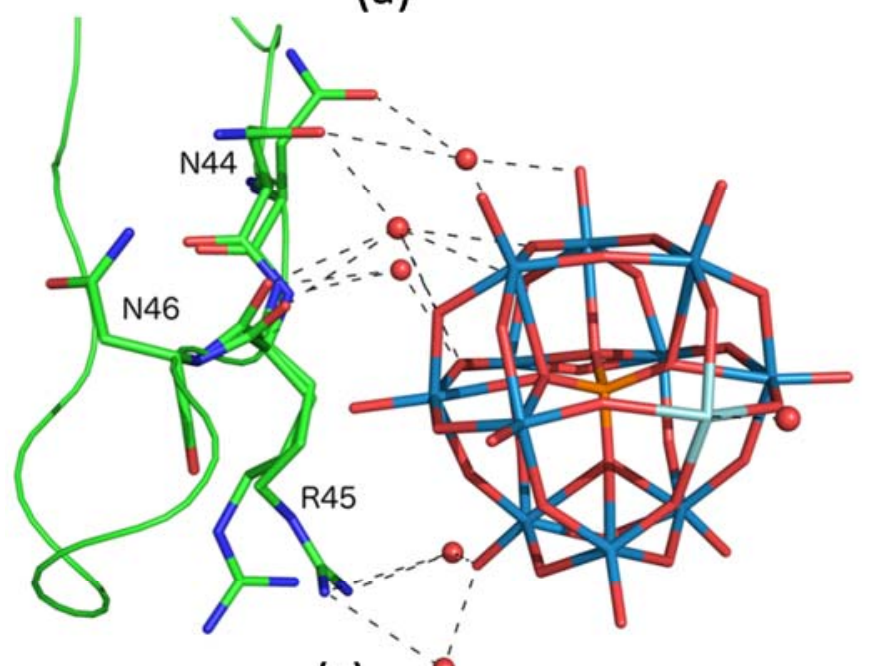

(c)

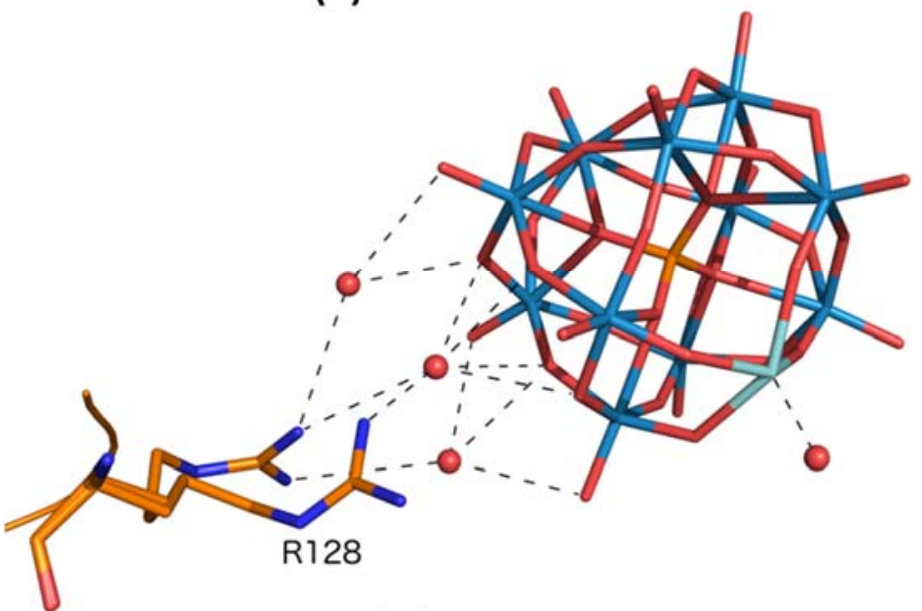

(e)

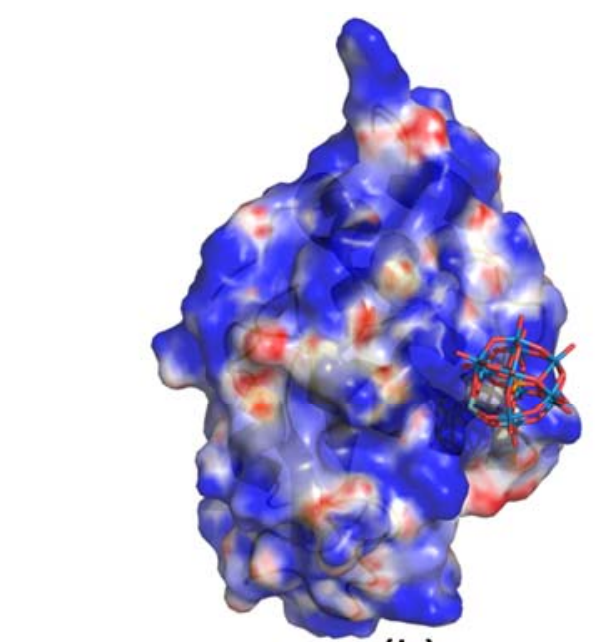

(b)
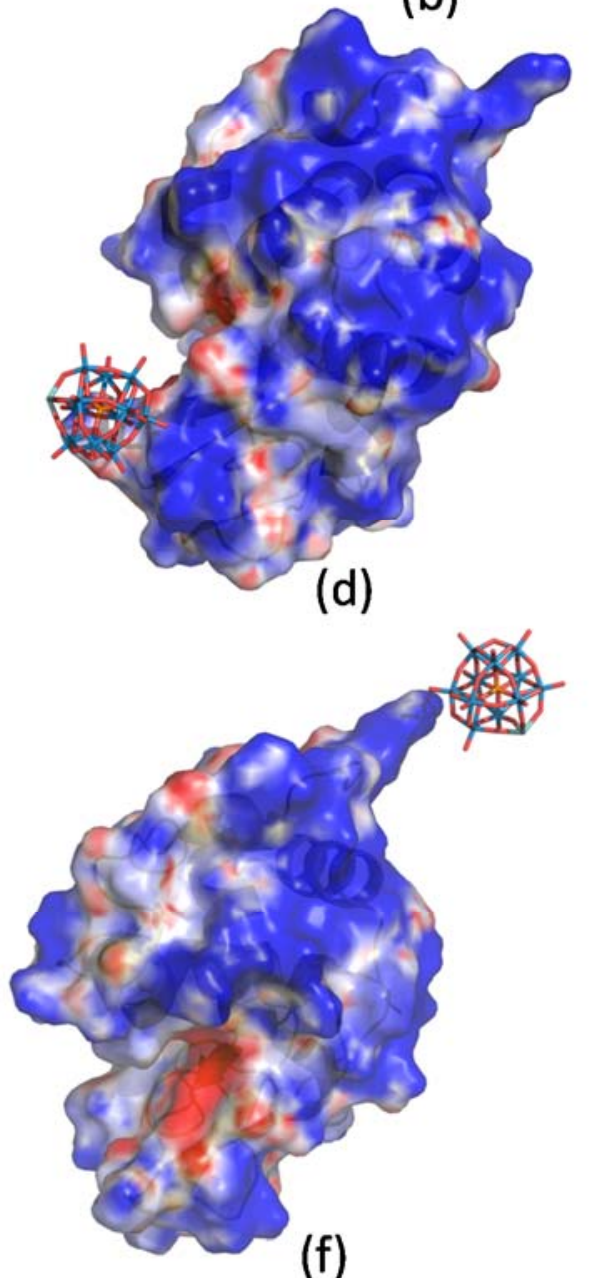

(f)

Figure 2: Three binding sites between 1 and HEWL. Water molecules are depicted as red spheres. Zirconium is colored in pale cyan, tungsten atoms in blue and the phosphorous atom in orange. a, c, e) $\mathrm{H}$-bonding interactions (dashes) between oxygen atoms of 1 with the following three binding sites of HEWL: a) Gly16, Tyr20 and Arg21, c) Asn44, Arg45 and Asn46, and e) Arg128 respectively. b, d, f) \pm 5 kT/e electrostatic potential maps of HEWL plotted on the van der Waals surface, generated using the PDB2PQR webtool[19] and the APBS plugin in Pymo|[20] with 1 displayed in proximity of b) Tyr20 and Arg21, d) Asn44, Arg45 and Asn46 and f) Arg128. 
Solution NMR experiments of the crystallization mixture confirmed that the folded HEWL structure was preserved upon binding to 2 (see Figure S6). In the presence of 2, the ${ }^{1} \mathrm{H}$ NMR spectrum of HEWL was very similar to that of native HEWL, where the aliphatic peaks seen below 0 ppm and the well spread amide resonances between 6 and 10 ppm were indicative of a folded protein structure.

${ }^{31} \mathrm{P}$ NMR titration experiments were performed to confirm that interaction takes place in solution and to observe the effect of HEWL on possible POM equilibria. The ${ }^{31} \mathrm{P}$ NMR experiments indicated only the presence of $2(-14.65 \mathrm{ppm}$, $-14.74 \mathrm{ppm}$ ) and the $\alpha$-lacunary Keggin POM (-10.71 ppm) upon addition of HEWL (Figure S7). The proposed catalyst in the $\mathrm{x}$-ray structure is the monomeric species 1 which is not observed in the ${ }^{31} \mathrm{P}$ NMR spectrum. We therefore hypothesize that the addition of increasing amounts of HEWL catalyzes the dissociation of the dimeric POM species 2 into the monomeric species 1 and the $\alpha$-lacunary species. We propose that 1 then forms a large complex with HEWL, causing the disappearance of the ${ }^{31} \mathrm{P}$ signals of $\mathbf{2}$. As the dissociation of $\mathbf{2}$ into $\mathbf{1}$ is very likely catalysed by the addition of HEWL, we therefore believe that $\mathbf{1}$ is the net catalyst inducing hydrolysis of HEWL. Disappearance of the ${ }^{31} \mathrm{P}$ NMR signals upon addition of increasing concentrations of protein is a common phenomenon observed in ligand/protein binding studies. ${ }^{[21]}$ It is induced by formation of a large POM/protein complex that has a long correlation time and therefore a fast relaxation. Disappearance of ${ }^{31} \mathrm{P}$ NMR signals instead of the appearance of new peaks suggests that an exchange binding mechanism takes place. ${ }^{[21]}$ Similarly to previous NMR studies, there was no evidence for the presence of free $1: 1 \mathrm{Zr}$ Keggin complex (1), however, the existence of this species has been recently suggested by DOSY NMR studies, which identified a fast equilibrium between a 2:2 $\mathrm{Zr}$-Keggin $\mathrm{POM}$, $\left[\left\{\alpha-\mathrm{PW}_{11} \mathrm{O}_{39} \mathrm{Zr}(\mu-\mathrm{OH})\left(\mathrm{H}_{2} \mathrm{O}\right)\right\}_{2}\right]^{8-}$ and its $1: 1$ form at $\mathrm{pD}$ 6.4. ${ }^{[22]}$ Furthermore, DFT theoretical calculations have also proven the possibility for the $1: 1$ species to exist in solution. ${ }^{[22]}$

In summary, monomeric Zr-Keggin POM (1) was successfully co-crystallized with HEWL and the x-ray structure shows three binding sites, of which two were located in close proximity to the previously established hydrolysis sites. The negatively charged Keggin POM electrostatically interacted with HEWL at all three positions with positively charged regions of HEWL. For the first time, structural and experimental evidence has been obtained for the existence of the monomeric Zr-Keggin complex (1), which is the presumed active catalyst in the hydrolysis of HEWL. Co-crystallization of catalytically active POMs with proteins offers unique insight into their exact binding mode at the molecular level. It also increases our understanding of the structure-activity relationship of protein hydrolysis by metal-functionalized POMs and may advance the application of metal-substituted POMs as a novel class of artificial proteases.

\section{Acknowledgements}

A.S. acknowledges FWO Flanders (Belgium) for a doctoral fellowship. E.D.Z. acknowledges IWT Flanders for a doctoral fellowship. We thank Dr. P. Legrand for assistance with the data collection at beamline Proxima-1 of the SOLEIL synchrotron facility. We thank Prof. M. De Maeyer and Drs. J. De Raeymaecker for calculating the volume of the voids.

Keywords: artificial enzymes $\bullet$ polyoxometalates $\bullet$ lysozyme $\bullet$ co-crystallization $\bullet$ Interaction mode

[1] a) A. Proust, R. Thouvenot, P. Gouzerh, Chemical Communications 2008, 1837-1852; b) J. T. Rhule, C. L. Hill, D. A. Judd, Chemical Reviews 1998, 98, 327-357; c) D.-L. Long, E. Burkholder, L. Cronin, Chemical Society Reviews 2007, 36, 105-121; d) D.-L. Long, R. Tsunashima, L. Cronin, Angewandte Chemie-International Edition 2010, 49, 1736-1758.

[2] Z. Guangjin, B. Keita, C. T. Craescu, S. Miron, P. de Oliveira, L. Nadjo, Journal of Physical Chemistry B 2007, 111, 11253-11259.

[3] K. Stroobants, E. Moelants, H. G. T. Ly, P. Proost, K. Bartik, T. N. Parac-Vogt, Chemistry-a European Journal 2013, 19, 2848-2858.

[4] A. Sap, G. Absillis, T. N. Parac-Vogt, Dalton Transactions 2015, 44, 1539-1548.

[5] a) K. Stroobants, G. Absillis, E. Moelants, P. Proost, T. Parac-Vogt, Chemistry-a European Journal 2014, 20, 3894-3897; b) K. Stroobants, V. Goovaerts, G. Absillis, G. Bruylants, E. Moelants, P. Proost, T. N. Parac-Vogt, Chemistry-a European Journal 2014, 20, 9567-9577.

[6] R. M. Smith, D. E. Hansen, Journal of the American Chemical Society 1998, 120, 8910-8913.

[7] a) L. Zhu, R. Bakhtiar, N. M. Kostic, Journal of Biological Inorganic Chemistry 1998, 3, 383-391; b) E. N. Korneeva, M. V. Ovchinnikov, N. M. Kostic, Inorganica Chimica Acta 1996, 243, 9-13.

[8] C. N. Kato, A. Shinohara, K. Hayashi, K. Nomiya, Inorganic Chemistry 2006, 45, 8108-8119.

[9] R. D. Peacock, T. J. R. Weakley, Journal of the Chemical Society A: Inorganic, Physical, Theoretical 1971, 1836-1839.

[10] a) M. A. Fedotov, B. Z. Pertsikov, D. K. Danovich, Polyhedron 1990, 9, 1249-1256; b) C. Zhang, R. C. Howell, K. B. Scotland, F. G. Perez, L. Todaro, L. C. Francesconi, Inorganic Chemistry 2004, 43, 7691-7701.

[11] R. Diamond, Journal of Molecular Biology 1974, 82, 371-\&.

[12] M. C. Vaney, S. Maignan, M. RiesKautt, A. Ducruix, Acta Crystallographica Section D-Biological Crystallography 1996, 52, 505-517.

[13] E. Krissinel, K. Henrick, in Computational Life Sciences, Proceedings, Vol. 3695 (Eds.: M. R. Berthold, R. Glen, K. Diederichs, O. Kohlbacher, I. Fischer), 2005, pp. 67-78.

[14] a) R. Ladenstein, A. Bacher, R. Huber, Journal of Molecular Biology 1987, 195, 751-753; b) J. Thygesen, S. Weinstein, F. Franceschi, A. Yonath, Structure 1996, 4, 513-518; c) G. Rudenko, L. Henry, C. Vonrhein, G. Bricogne, J. Deisenhofer, Acta Crystallographica Section D-Biological Crystallography 2003, 59, 1978-1986; d) M. Zebisch, M. Krauss, P. Schaefer, N. Straeter, Journal of Molecular Biology 2012, 415, 288-306; e) S. O. Dahms, M. Kuester, C. Streb, C. Roth, N. Straeter, M. E. Than, Acta Crystallographica Section D-Biological Crystallography 2013, 69, $284-297$.

[15] a) S. G. Mauracher, C. Molitor, R. Al-Oweini, U. Kortz, A. Rompel, Acta Crystallographica Section D-Biological Crystallography 2014, 70, 2301-2315; b) S. G. Mauracher, C. Molitor, R. Al-Oweini, U. Kortz, A. Rompel, Acta Crystallographica Section F-Structural Biology and Crystallization Communications 2014, 70, 263-266.

[16] a) F. Schluenzen, A. Tocilj, R. Zarivach, J. Harms, M. Gluehmann, D. Janell, A. Bashan, H. Bartels, I. Agmon, F. Franceschi, A. Yonath, Cell 2000, 102, 615-623; b) J. Harms, F. Schluenzen, R. Zarivach, A. Bashan, S. Gat, I. Agmon, H. Bartels, F. Franceschi, A. Yonath, Cell 2001, 107, 679-688.

[17] a) J. Schemberg, K. Schneider, U. Demmer, E. Warkentin, A. Mueller, U. Ermler, Angewandte Chemie-International Edition 2007, 46, 2408-2413; b) B. Kowalewski, J. Poppe, U. Demmer, E. Warkentin, T. Dierks, U. Ermler, K. Schneider, Journal of the American Chemical Society 2012, 134, 9768-9774.

[18] L. Schrödinger, The PyMOL Molecular Graphics System, Version 1.3r1 2010. 
[19] T. J. Dolinsky, J. E. Nielsen, J. A. McCammon, N. A. Baker, Nucleic Acids Research 2004, 32, W665-W667.

[20] N. A. Baker, D. Sept, S. Joseph, M. J. Holst, J. A. McCammon, Proceedings of the National Academy of Sciences 2001, 98, 10037-10041.

[21] O. Zerbe, in Methods and Principals in Medicinal Chemistry, Vol. 16 (Eds.: R. Mannhold, G. Folkers), Wiley VCH, Weinheim, 2003.

[22] T. K. N. Luong, P. Shestakova, T. T. Mihaylov, G. Absillis, K. Pierloot, T. N. Parac-Vogt, Chemistry A European Journal 2015, $21,1-13$. 


\section{Supplementary Information}

Table of contents

1. Experimental Section

2. X-ray data collection and structure refinement statistics

3. Hydrogen bonding interactions

4. NMR spectra of $\mathbf{2}$

5. Zr Keggin POM - HEWL co-crystals with precipitation

6. Electron density maps

7. Solution NMR experiments of the crystallization mixture

\section{References}

\section{Experimental Section}

Synthesis: $\left(\mathrm{Et}_{2} \mathrm{NH}_{2}\right)_{10}\left[\mathrm{Zr}\left(\mathrm{PW}_{11} \mathrm{O}_{39}\right)_{2}\right] .7 \mathrm{H}_{2} \mathrm{O}(\mathrm{Zr} 1-\mathrm{K} 2)(2)$ was synthesized according to the published procedure and characterized by ${ }^{31} \mathrm{P}$ NMR spectroscopy. ${ }^{[1]}$ Hen egg-white lysozyme (HEWL) was obtained from Sigma-Aldrich in the highest available purity and was used without any further purification.

Co-crystallization: Crystals of HEWL and the 1:2 Zr-substituted Keggin $\mathrm{POM},\left(\mathrm{Et}_{2} \mathrm{NH}_{2}\right)_{10}\left[\mathrm{Zr}\left(\mathrm{PW}_{11} \mathrm{O}_{39}\right)_{2}\right] .7 \mathrm{H}_{2} \mathrm{O}(\mathbf{2})$, were grown in a period of three weeks using the sitting drop vapour diffusion method. In the drop, $1 \mu \mathrm{HEWL}(3.5 \mathrm{mM}$, dissolved in $10 \mathrm{mM}$ phosphate buffer $\mathrm{pH}$ 7.4), was mixed with $1 \mu \mathrm{l}(2)(3.5 \mathrm{mM}$, dissolved in $10 \mathrm{mM}$ phosphate buffer $\mathrm{pH} 7.4$ containing $5 \% \mathrm{DMSO})$ and $1 \mu \mathrm{l}$ precipitant solution (2.0 M ammonium sulphate, $0.1 \mathrm{M}$ sodium HEPES pH 7.5). Before cryofreezing, the crystals (with an approximate size of 0.1-0.2 mm in length) were soaked in a $20 \%$ PEG 400 solution as cryoprotectant.

Structure determination and refinement: X-ray data were collected on a Pilatus $6 \mathrm{M}$ detector using a wavelength of $0.8856 \AA$ and a temperature of $100 \mathrm{~K}$ at the Proxima1 beamline at the Soleil synchrotron (Paris, France) after an X-ray fluorescence spectrum proved the presence of $\mathrm{Zr}$ and $\mathrm{W}$ in the crystal. Data analysis and reduction were performed using XDS v. Januari 10, $2014{ }^{22]}$, Phenix. merging statistics v.1.9, Phenix. Reflection file editor v.1.9. ${ }^{[3]}$ Phases were calculated using Phaser v.2.5.6 ${ }^{[4]}$ and using HEWL (PDB ID: 1LYZ, ${ }^{[5]}$ ) as phasing model. The structure was refined using Phenix. refine v.1.9 ${ }^{\left[{ }^{6]}\right.}$ and COOT v.0.7.2 ${ }^{[7]}$, each cycle consisting of bulk-solvent and anisotropic scaling, individual coordinate and B-factor refinement. After the first refinement cycle the tungsten atoms and the zirconium atom of the 1:1 Zr-substituted Keggin POM (1) appeared very clear in the electron density maps, with zirconium having a reduced electron density compared to the tungsten atoms (see Figure S4). The occupancy of 1 and residues with alternative configurations were refined until convergence. Throughout refinement, reasonable electron densities (both in the $2 \mathrm{mFo-DFc}$ and $\mathrm{mFo}-\mathrm{DFc}$ map) as well as reasonable B-factors and interaction partners were required to add ligands (ethylene diamine and dimethyl sulfoxide) and water molecules to the model. Weak electron density indicated a water molecule coordinating to the zirconium atom of 1 and further refined with the same occupancy as 1 (see Figure S5). Based on the electron density maps, this is the best model we can refine for the POM, although it is not excluded that further water molecules coordinate to Zr. Restraints files for the ligands were generated using eLBOW. ${ }^{[8]}$ As there was no restraints cif-file available for this metal-substituted POM, we manually generated one based on the available structure of 2 in the Cambridge Structural Database ${ }^{[9]}$ (Refcode: XEQFAB ${ }^{[1]}$ ). The data collection and refinement statistics can be found in Table S1. The structure was deposited in the Protein DataBank and has the following code: 4XYY.

${ }^{31} \mathrm{P}$ NMR spectroscopy: Solutions containing $\left(\mathrm{Et}_{2} \mathrm{NH}_{2}\right)_{10}\left[\mathrm{Zr}\left(\mathrm{PW}_{11} \mathrm{O}_{39}\right)_{2}\right] .7 \mathrm{H}_{2} \mathrm{O}(2 \mathrm{mM})$ in the absence and presence of increasing concentrations of HEWL $(0 \mathrm{mM}, 0.25 \mathrm{mM}, 0.5 \mathrm{mM}, 1 \mathrm{mM})$ were prepared in phosphate buffer $\left(\mathrm{pH} 7.4,10 \% \mathrm{D}_{2} \mathrm{O}\right)$. ${ }^{31} \mathrm{P}$ NMR spectra were recorded on a Bruker Avance $600 \mathrm{MHz}$ spectrometer directly after mixing. As an external reference, $25 \% \mathrm{H}_{3} \mathrm{PO}_{4}$ in $\mathrm{D}_{2} \mathrm{O}$ in a sealed capillary was used.

${ }^{1} \mathrm{H}$ NMR spectroscopy: Solutions containing HEWL $(1 \mathrm{mM})$ in the absence or presence of $10 \%$ SDS, DTT (100 mM) / $\left(\mathrm{Et}_{2} \mathrm{NH}_{2}\right)_{10}\left[\mathrm{Zr}\left(\mathrm{PW}_{11} \mathrm{O}_{39}\right)_{2}\right] .7 \mathrm{H}_{2} \mathrm{O}(0.1 \mathrm{mM})$ were prepared in phosphate buffer $\left(\mathrm{pH} 7.4,10 \% \mathrm{D}_{2} \mathrm{O}\right) .{ }^{1} \mathrm{H}$ NMR spectra were recorded on a Bruker Avance $600 \mathrm{MHz}$ spectrometer directly after mixing. Tetramethylsilane (TMS) was used as an internal reference. 


\section{X-ray data collection and structure refinement statistics}

Table S1: X-ray data collection and structure refinement statistics for the co-crystal of HEWL - 1:1 Zr-Keggin POM.

\begin{tabular}{|c|c|}
\hline Parameter & Zr-Keggin POM - HEWL \\
\hline Space group & $\mathrm{P} 4{ }_{3} 212$ \\
\hline \multicolumn{2}{|l|}{ Unit cell parameters } \\
\hline$a, b, c(\AA)$ & $77.23,77.23,37.96$ \\
\hline Resolution range ${ }^{[\mathrm{a}]}(\AA)$ & $38.62-1.38(1.43-1.38)$ \\
\hline $\mathrm{R}_{\text {merge }}(\%)$ & $11.3(69.5)$ \\
\hline$<I / \sigma(I)>$ & $10.2(2.0)$ \\
\hline No. of unique reflections & $45045(4502)$ \\
\hline Multiplicity & $7.6(7.3)$ \\
\hline Completeness (\%) & $99.8(99.3)$ \\
\hline $\mathrm{R}_{\text {work }} / \mathrm{R}_{\text {free }}[\mathrm{b}](\%)$ & $16.18 / 18.37$ \\
\hline \multicolumn{2}{|l|}{ RMSD from ideal } \\
\hline Bond lengths $(\AA)$ & 0.009 \\
\hline Bond angles $\left({ }^{\circ}\right)$ & 2.040 \\
\hline \multicolumn{2}{|c|}{ Average isotropic $B$-factors $\left(\AA^{2}\right)$} \\
\hline Main chain & 17.92 \\
\hline Side chain & 21.88 \\
\hline Water molecules & 31.20 \\
\hline Ligands & 27.79 \\
\hline 1:1 Zr-Keggin POM & 16.59 \\
\hline
\end{tabular}

[a] Values in parentheses are for the highest resolution shell.

[b] $R_{\text {free }}$ is calculated using a random $5 \%$ of data excluded from the refinement.

\section{Hydrogen bonding interactions}

Table S2: Hydrogen bonding interactions between 1:1 Zr-Keggin POM and HEWL as depicted in Figure 2a.

\begin{tabular}{llll} 
Atom of 1:1 Zr-Keggin POM & HEWL interaction partner & Type & Distance* $(\AA)$ \\
\hline \multirow{2}{*}{ O52 } & Y20 [OH] & Direct & 2.8 \\
& Y20 [OH] & Water mediated & $3.1 / 3.4$ \\
& R21/A [NH1] & Water mediated & $3.1 / 3.0$ \\
& R21/A [N] & Water mediated & $3.0 / 3.0$ \\
& R21/B [N] & Water mediated & $3.0 / 3.0$ \\
& S100 [OG] & Water mediated & $3.1 / 2.9$ \\
O54 & K96 [NZ] & Direct & 2.6 \\
& G16 [O] & Water mediated & $3.0 / 2.8$ \\
O72 & G16 [O] & Water mediated & $2.9 / 2.8$ \\
& G16 [O] & Water mediated & $3.2 / 2.8$
\end{tabular}

* In case of water mediated interactions, the distance from the specified atom of 1:1 Zr-Keggin POM is followed by the distance between the water molecule and the HEWL interaction partner. 
Table S3: Hydrogen bonding interactions between 1:1 Zr-Keggin POM and HEWL as depicted in Figure 2c. All interactions are water mediated.

\begin{tabular}{|c|c|c|}
\hline Atom of 1:1 Zr-Keggin POM & HEWL interaction partner & Distance $^{*}(\AA)$ \\
\hline \multirow[t]{3}{*}{ O48 } & R45/B [NH1] & $2.6 / 3.2$ \\
\hline & R45/B [NH1] & $2.6 / 3.4$ \\
\hline & R45/B [NH2] & $2.6 / 2.7$ \\
\hline \multirow[t]{3}{*}{ O51 } & N44/B [NH1] & $3.1 / 2.9$ \\
\hline & R45/A [O] & $3.1 / 3.0$ \\
\hline & R45/B [O] & $3.1 / 3.1$ \\
\hline \multirow[t]{3}{*}{$\mathrm{O} 64$} & N44/B [OD1] & $3.2 / 2.9$ \\
\hline & R45/A [O] & $3.2 / 3.0$ \\
\hline & R45/B [O] & $3.2 / 3.1$ \\
\hline \multirow[t]{3}{*}{$\mathrm{O} 65$} & N44/B [OD1] & $3.2 / 2.9$ \\
\hline & R45/A [O] & $3.2 / 3.0$ \\
\hline & R45/B [O] & $3.2 / 3.1$ \\
\hline \multirow[t]{2}{*}{$\mathrm{O} 70$} & R45/A [N] & $2.8 / 2.8$ \\
\hline & R45/B [N] & $2.8 / 3.0$ \\
\hline \multirow[t]{5}{*}{071} & N44/A [OD1] & $3.0 / 3.1$ \\
\hline & N44/B [OD2] & $3.0 / 3.4$ \\
\hline & N44/B [OD1] & $3.3 / 2.9$ \\
\hline & R45/A [O] & $3.3 / 3.0$ \\
\hline & R45/B [O] & $3.3 / 3.1$ \\
\hline \multirow[t]{2}{*}{$\mathrm{O} 72$} & N44/A [OD1] & $2.7 / 3.1$ \\
\hline & N44/B [OD1] & $2.7 / 3.4$ \\
\hline
\end{tabular}

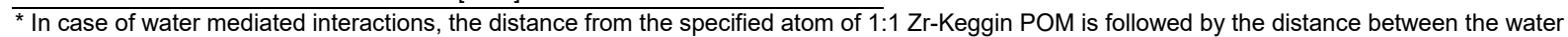
molecule and the HEWL interaction partner.

Table S4: Hydrogen bonding interactions between 1:1 Zr-Keggin POM and HEWL as depicted in Figure 2e. All interactions are water mediated.

\begin{tabular}{lll} 
Atom of 1:1 Zr-Keggin POM & HEWL interaction partner & Distance* $^{*}()$ \\
\hline O54 & R128/A [NH2] & $3.0 / 3.4$ \\
O55 & R128/A [NH2] & $2.9 / 3.1$ \\
& R128/B [NH2] & $2.9 / 2.2$ \\
O66 & R128/A [NH2] & $2.9 / 3.4$ \\
& R128/A [NH2] & $3.1 / 3.1$ \\
& R128/B [NH2] & $3.1 / 2.2$ \\
O72 & R128/A [NH2] & $3.2 / 3.4$ \\
& R128/A [NH1] & $2.8 / 3.4$ \\
& R128/A [NH1] & $3.2 / 3.1$ \\
O74 & R128/B [NH2] & $3.2 / 2.2$ \\
\hline
\end{tabular}

* In case of water mediated interactions, the distance from the specified atom of $1: 1 \mathrm{Zr}$-Keggin POM is followed by the distance between the water molecule and the HEWL interaction partner. 


\section{4. $\quad$ NMR spectra of 2}

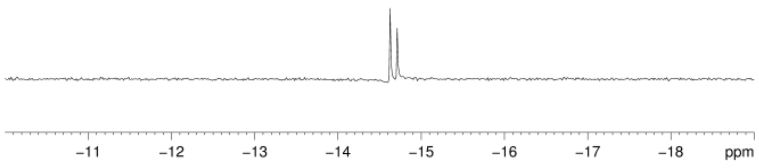

Figure S1: ${ }^{31} \mathrm{P}$ NMR spectrum of $2(2.0 \mathrm{mM})$ in $100 \% \mathrm{D}_{2} \mathrm{O}$. No pD adjustments were made. Only the dimeric species of 2 is present with characteristic NMR signals at $-14.63 \mathrm{ppm}$ and $-14.71 \mathrm{ppm}$.

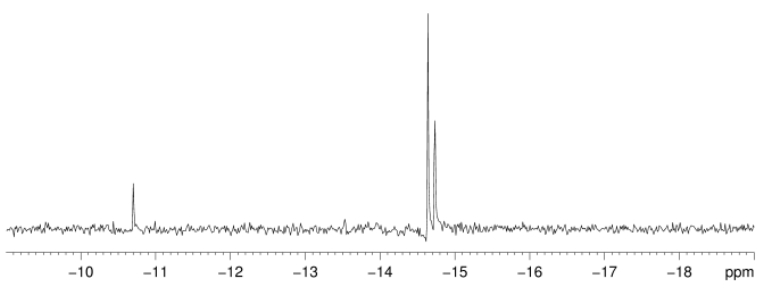

Figure S2: ${ }^{31} \mathrm{P}$ NMR spectrum of $2(2.0 \mathrm{mM})$ in $90 \%$ phosphate buffer, $10 \% \mathrm{D}_{2} \mathrm{O}$ at pH 7.4. 2 is present in its dimeric species with characteristic signals at -14.64 and $-14.73 \mathrm{ppm}$, and the NMR signal at $-10.70 \mathrm{ppm}$ represents the $\alpha$-lacunary species.

\section{Zr Keggin POM - HEWL co-crystals with precipitation}

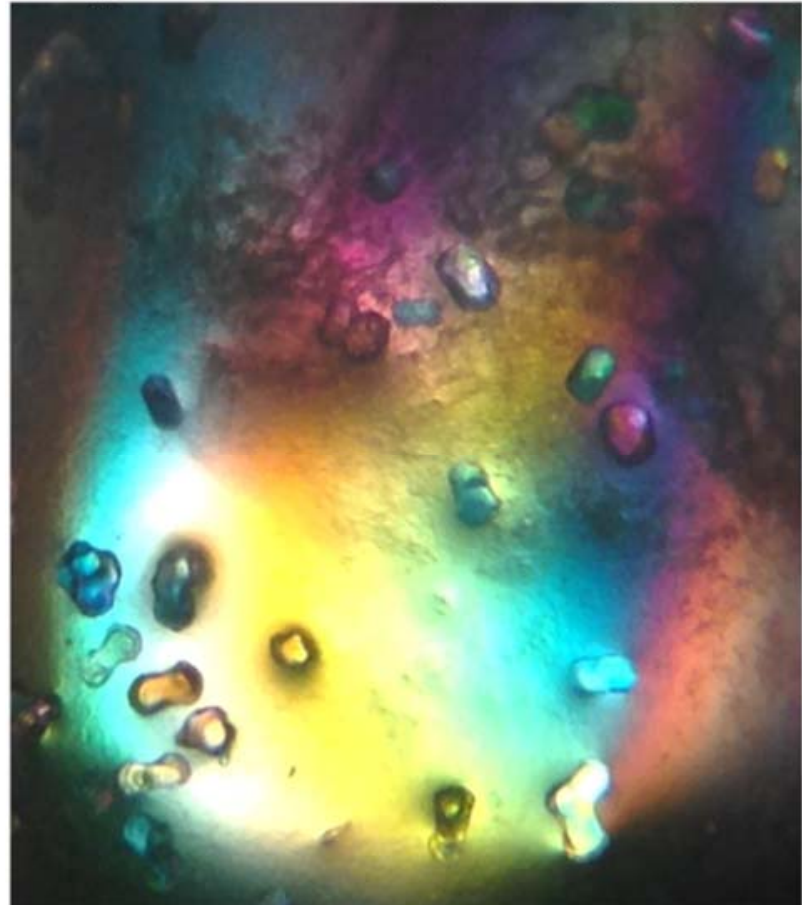

Figure S3: Zr Keggin POM - HEWL co-crystals. Even though the molar ratio of Zr Keggin : HEWL in the drop was 1:1, $100 \%$ occupancy of 1 cannot be expected as the POM partially precipitates. 


\section{Electron density maps}

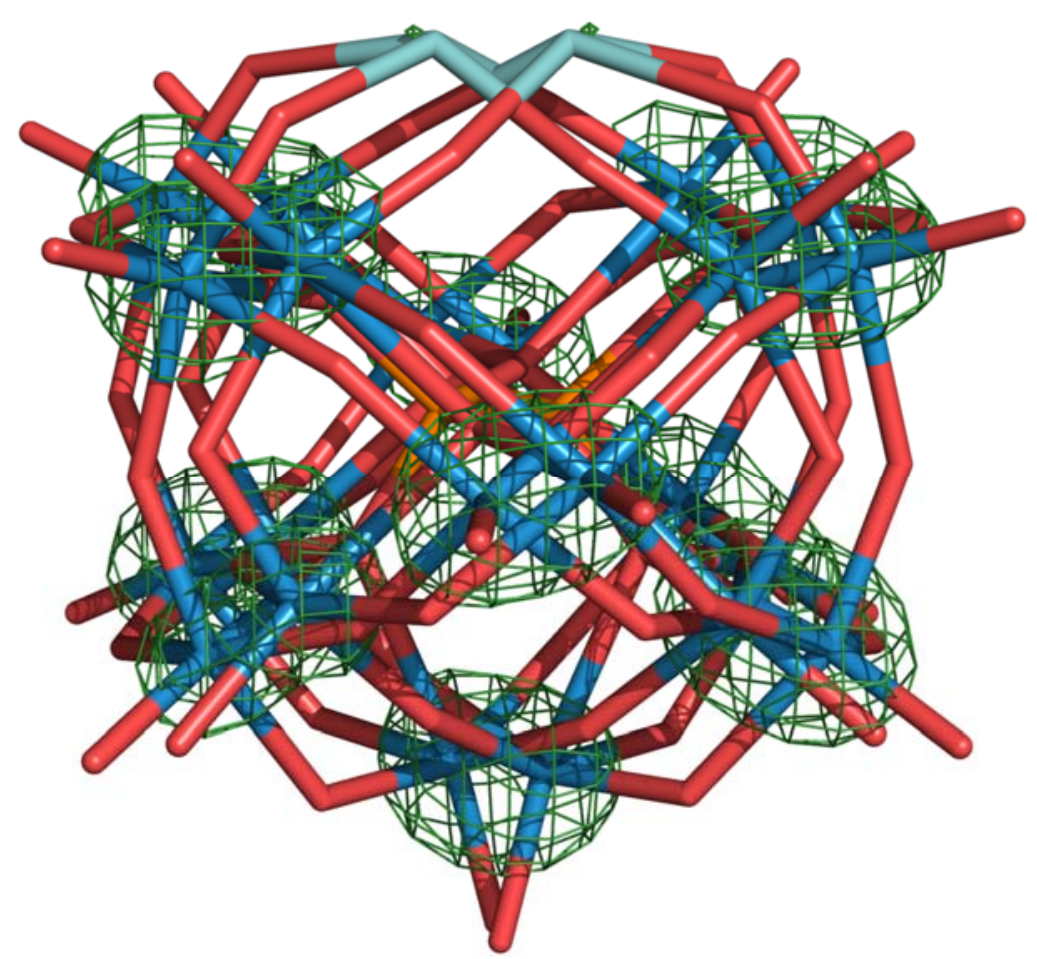

Figure S4: Fo-Fc electron density map before addition of 1 showing the lower density of zirconium at a $\sigma$-level of 5.0 , while tungsten atoms are still nicely contoured.

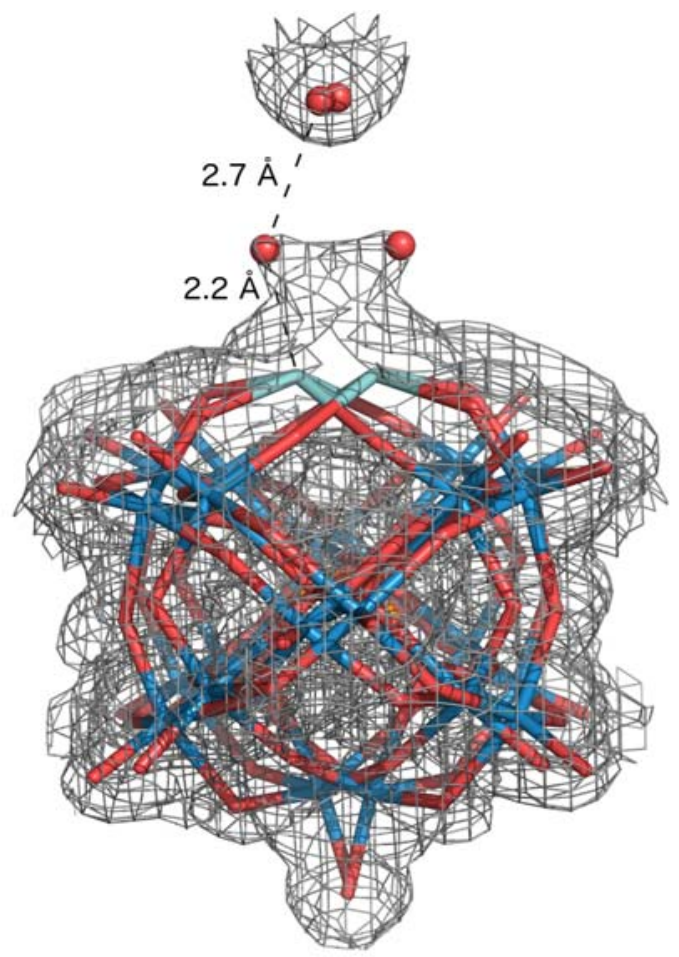

Figure S5: Final 2Fo-Fc map showing the two extra water molecules and symmetry mates (contoured at $\sigma$-level $=0.0$ ). 


\section{Solution NMR experiments of the crystallization mixture}

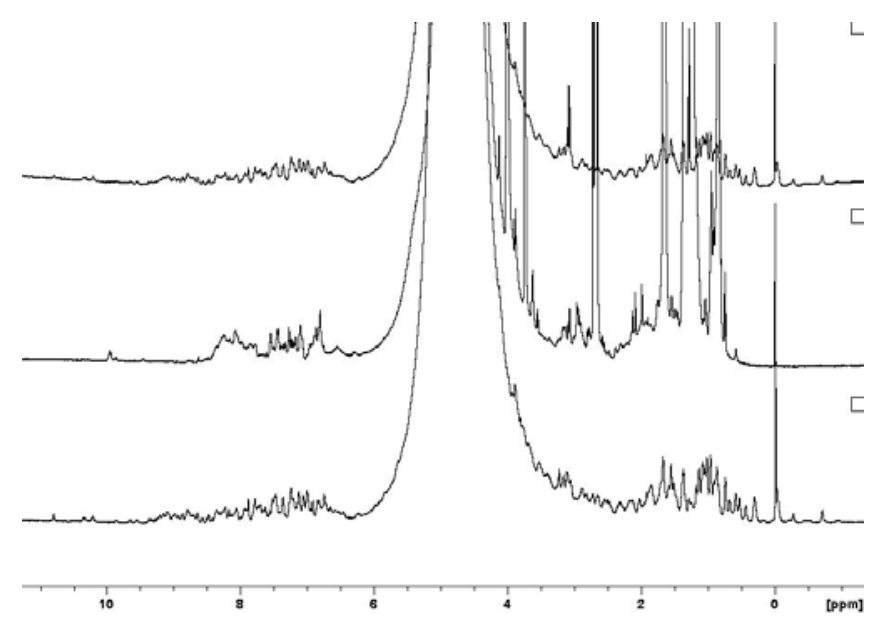

Figure S6: ${ }^{1} \mathrm{H}$ NMR spectrum of HEWL (bottom), in the presence of SDS/DTT (middle), and in the presence of 2 (top). Addition of the disulfide reducing agent, dithiothreitol (DTT), and the anionic detergent, sodium dodecyl sulfate (SDS), results in the disappearance of aliphatic peaks at low $\delta$ values and a change in amide resonances, indicating protein denaturation.
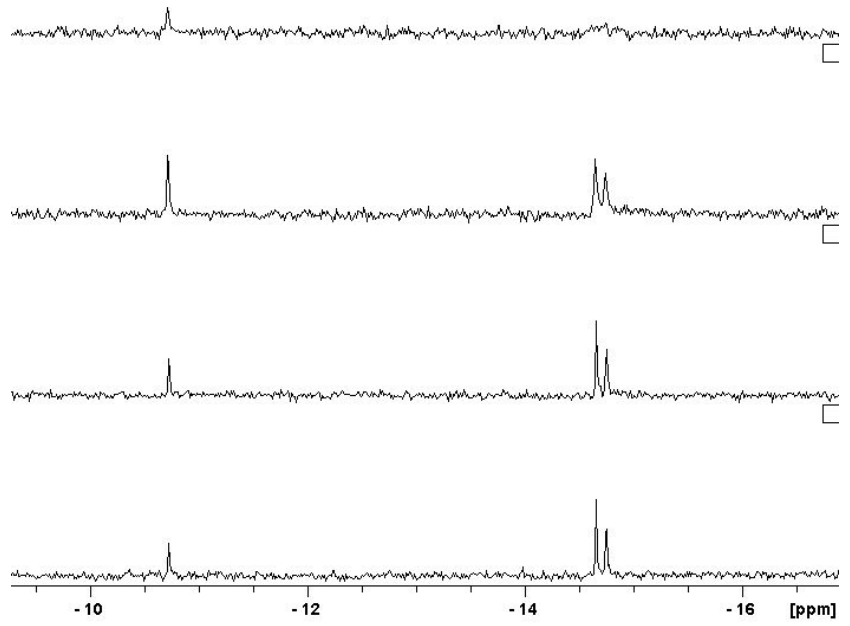

Figure S7: ${ }^{31} \mathrm{P}$ NMR spectrum of 2 in the absence and presence of increasing equivalents of HEWL. From bottom to top: $0,0.125,0.250,0.500$ equivalents of $\mathrm{HEWL}$ were added.

\section{References}

C. N. Kato, A. Shinohara, K. Hayashi, K. Nomiya, Inorganic Chemistry 2006, 45, 8108-8119.

W. Kabsch, Acta Crystallographica Section D-Biological Crystallography 2010, 66, 125-132.

P. D. Adams, P. V. Afonine, G. Bunkoczi, V. B. Chen, I. W. Davis, N. Echols, J. J. Headd, L.-W. Hung, G. J. Kapral, R. W. Grosse-Kunstleve, A. J. McCoy, N. W. Moriarty, R. Oeffner, R. J. Read, D. C. Richardson, J. S. Richardson, T. C. Terwilliger, P. H. Zwart, Acta Crystallographica Section D-Biological Crystallography 2010, 66, 213-221.

[4] A. J. McCoy, R. W. Grosse-Kunstleve, P. D. Adams, M. D. Winn, L. C. Storoni, R. J. Read, Journal of Applied Crystallography 2007, 40, 658-674.

[5] R. Diamond, Journal of Molecular Biology 1974, 82, 371-\&.

[6] P. V. Afonine, R. W. Grosse-Kunstleve, N. Echols, J. J. Headd, N. W. Moriarty, M. Mustyakimov, T. C. Terwilliger, A. Urzhumtsev, P. H. Zwart,

P. D. Adams, Acta Crystallographica Section D-Biological Crystallography 2012, 68, 352-367.

P. Emsley, B. Lohkamp, W. G. Scott, K. Cowtan, Acta Crystallographica Section D-Biological Crystallography 2010, 66, 486-501.

N. W. Moriarty, R. W. Grosse-Kunstleve, P. D. Adams, Acta Crystallographica Section D-Biological Crystallography 2009, 65, 1074-1080.

F. Allen, Acta Crystallographica Section B 2002, 58, 380-388. 